\title{
Endokarditisprophylaxe: Paradigmenwechsel gerechtfertigt
}

\section{Die Empfehlung zum Verzicht auf eine Endokarditisprophylaxe bei invasiven Eingriffen ist weitgehend befolgt worden und hat nicht zu einer Zunahme der Erkrankungen geführt.}

- Das nationale Institute of Health and Clinical Excellence in England hat im März 2008 empfohlen, Patienten mit Risiko für eine Endokarditis bei invasiven zahnärztlichen und anderen Eingriffen keine Antibiotika mehr prophylaktisch $\mathrm{zu}$ verabreichen. Die Autoren haben nun aus den nationalen Registern für den Zeitraum zwischen Januar 2000 und Mai 2010 die Zahlen der für die Prophylaxe verordneten Antibiotika und der Endokarditiserkrankungen entnommen.

Danach nahmen die Verschreibungen für Antibiotika zur Endokarditisprophylaxe ab März 2008 innerhalb weniger Monate dramatisch ab. Über-

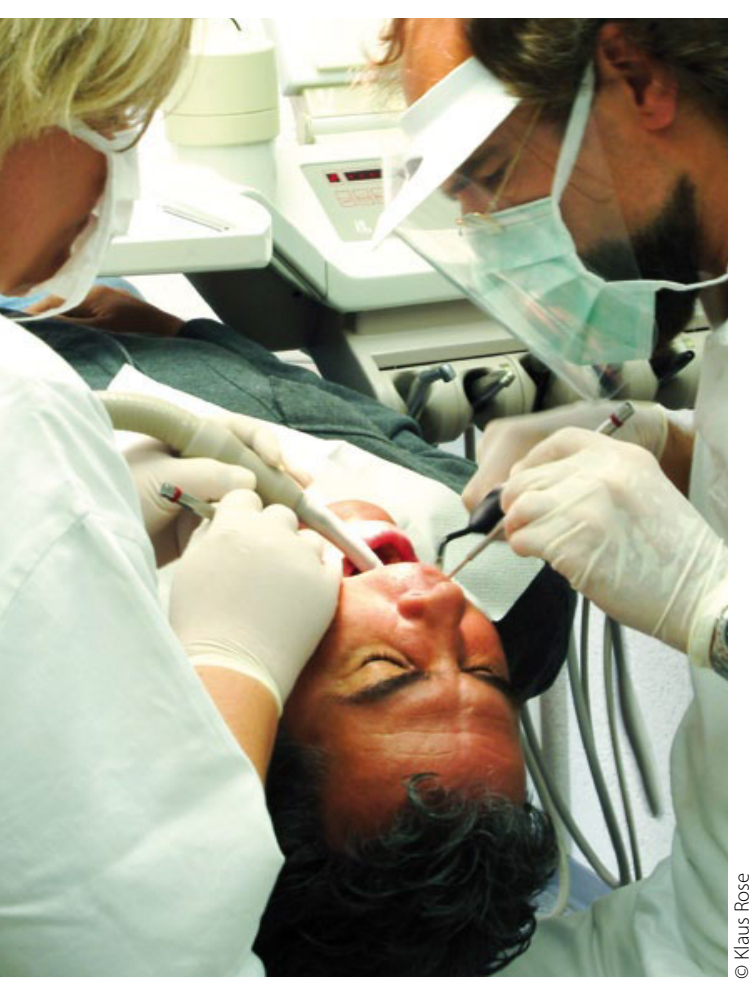

Wird das Myokarditisrisiko beim Zahnarzt überschätzt? wiegend muss es sich um zahnärztliche Eingriffe gehandelt haben, denn die überwiegende Mehrzahl der Verordner waren Zahnärzte. Im Vergleich der zwölf Monate vor der Empfehlung zu 14 bis 25 Monaten danach gingen die Verordnungen von monatlich 10727 auf 2292, also um 78,6\% zurück. Die Rate der monatlichen Endokarditiserkrankungen blieb demgegenüber in diesem Zeitraum konstant und zeigt auch im Langzeitverlauf von Januar 2000 bis April 2010 lediglich eine konstant leicht ansteigende Gerade.

\section{Kommentar}

Die infektiöse Endokarditis ist eine seltene Erkrankung mit hoher Morbidität und Mortalität. Weil bei zahlreichen invasiven Eingriffen von der Zahnextraktion über die Herzkatheteruntersuchung bis zur Koloskopie Bakterien in die Blutbahn gelangen, galt bis zum März 2008 in England - und vielen anderen Ländern - die Empfehlung, Patienten mit erhöhtem Risiko für eine Endokarditis prophylaktisch Antibiotika zu geben, z.B. eine einzelne orale Dosis von $3 \mathrm{~g}$ Amoxicillin oder 600 mg Clindamycin. Allerdings gab es für die Wirksamkeit dieser Maßnahme keine zuverlässigen Evidenzen. Einige Studien stützen vielmehr die Vermutung, dass zahnärztliche invasive Eingriffe nicht Ursache für eine Endokarditis sind. Inzwischen kann man von einem Paradigmenwechsel sprechen, denn zahlreiche Fachgesellschaften weltweit haben vor einigen Jahren ihre Empfehlungen zur Endokarditisprophylaxe widerrufen. Sie bleibt heute nur noch bestimmten Hochrisikopatienten, vor allem solchen mit künstlichen oder rekonstruierten Herzklappen und mit bestimmten Herzfehlern vorbehalten.

H. HolzGReVE =

- M. H. Thornhill et al.: Impact of the NICE guideline recommending cessation of antibiotic prophylaxis for prevention of infectious endocarditis: before and after study. Brit. Med. J. 342 (2011) 1136 (Langfassung) 